Artículo

\title{
Una experiencia docente de la web 2.0 mediante el uso de blogs con estudiantes de educación de la Universidad Nacional, Heredia
}

\author{
A teaching experience of the web 2.0 through the use of blogs \\ with education students of the National University, Heredia \\ Manuel Chacón Ortiz* \\ mchacono@uned.ac.cr
}

ISSN 1996-1642, Editorial Universidad Don Bosco, año 11, No.19, enero-junio de 2017, pp. 51-67

Recibido: 29 de noviembre de 2016. Aprobado: 9 de enero de 2017

\begin{abstract}
Resumen
Se presentan los resultados de una evaluación sobre el uso de los blogs por parte de estudiantes de Pedagogía | y || Ciclo, Preescolar y Educación Especial de la Universidad Nacional (de Costa Rica). Se utilizó el blog con el fin de crear contenidos sobre los temas del curso Educación para la salud y calidad de vida. Los estudiantes debían realizar intervenciones considerando información que podría llegar a ser de interés por personas a cargo de niños y niñas en edad escolar. Participaron 41 estudiantes, 39 mujeres y 2 hombres. El estudio, de tipo cuantitativo, aplicó el cuestionario para la recolección de la información al finalizar cada periodo académico del año 2014 y 2015. Los resultados evidenciaron que los estudiantes nunca habían empleado el blog anteriormente; sin embargo, la experiencia resultó positiva y potenció nuevas habilidades y destrezas educativas y tecnológicas. Asimismo, el estudio muestra que existe empatía por el empleo de estas herramientas.
\end{abstract}

Palabras clave:Género, educación, TIC, blog, aprendizaje, evaluación, tecnología, mujeres.

\begin{abstract}
This paper analyzes the results of an evaluation of the use of blogs carried out with students of Education Sciences. A blog was used in order to create content on the topics of the class "Educación para la salud y la calidad de vida" The students were given instructions that information they write could become of interest for dependents of children of school age. In total, 41 students participated, 39 women and 2 men. The study is quantitative; a questionnaire for collecting information at the end of each academic period (2014 and 2015) was used. The results showed that the students had never used a blog before; however, the experience was positive enhancing new educational and technological skills. Also, the study shows that there is willingness to use these tools in education. In conclusion, universities may support the blog as an academic tool in higher education. Blogs allows future teachers to incorporate new cognitive and technological skills.
\end{abstract}

Keywords: Gender, education, ICT, blog, learning, evaluation, technology, women.

*Profesor de Investigación Educativa, Centro de Investigaciones en Educación - CINED, Escuela de Ciencias de la Educación, Universidad Estatal a Distancia, Costa Rica, 


\section{Introducción}

Hablar de tecnología y educación es necesariamente referirse a una relación continua. Actualmente, la tecnología se encuentra en todos los ámbitos de la vida. Es preciso idealizar menos las Tecnologías de la Comunicación e Información (TIC) y dar paso a su uso, reflexionar y rendir cuentas sobre los alcances y limitaciones derivados de su aplicación en la enseñanza.

Desde hace años, se debate el papel de la educación en una sociedad donde el eje de la producción es el conocimiento (Castells, 2006). Una de las acciones por realizar para alcanzar los objetivos del Marco de Acción de Dakar de la UNESCO (2000) es "aprovechar las nuevas tecnologías de la información y la comunicación para contribuir al logro de los objetivos de la educación para todos" (p. 9). Se debe evidenciar cómo las acciones en las cuales se integran las tecnologías apoyan o limitan el acceso a la educación y a la generación de nuevos conocimientos.

El país invierte una cantidad importante de recursos públicos para dotar y capacitar escuelas y colegios con tecnología en el sistema educativo (Programa Estado de la Nación, 2015). No obstante, este mismo informe evidencia deficiencias en la formación y el aprovechamiento de la tecnología. Por ejemplo, en los nuevos programas de matemática se mostró que los docentes, a pesar de conocer el uso de las tecnologías, las empleaban de forma deficiente, o los recursos eran subutilizados, se indicaba además que tales esfuerzos "son insuficientes si el docente no posee el hábito de aprovecharlas" (Programa Estado de la Nación, 2015, p. 161).

El aprendizaje de las tecnologías va más allá de una asignatura, del currículo, o de un programa de estudio, no existe forma de crear hábitos y buenas prácticas en el uso de las TIC si no se incorporan de forma constante en la enseñanza.

Las TIC son una oportunidad para el aprendizaje colaborativo, tanto entre escolares, adultos, como entre docentes y estudiantes, o a la inversa. Se señala que es responsabilidad de los maestros valorar y determinar cuáles son las herramientas tecnológicas más apropiadas para así emplear las que sirven con fines pedagógicos, críticamente contemplados y pedagógicamente orientados (Kalaš, 2010).

Por las razones anteriores, las experiencias y conocimientos que adquieren las estudiantes en su proceso de formación son indispensables tanto es aspectos operativos (software, gamificación, Web 2.0, entre otras) como en sus dimensiones técnicas (cámaras digitales, teléfonos inteligentes, portátiles, microscopios, robótica, etcétera).

Una experiencia docente de la web 2.0 mediante el uso de blogs con estudiantes de educación de la Universidad

52. Nacional, Heredia
Se critican frecuentemente los sistemas educativos, en todos los niveles, por la pedagogía que no ha logrado adaptar a los cambios tecnológicos propios del siglo. Aun se observan escuelas preocupadas por "transferir" o "depositar" información en los alumnos. Las instituciones educativas, para bien o para mal, 
en aspectos evaluativos y jerárquicos no se adaptan o no logran integrar la tecnología digital (Selwyn, 2014).

El Ministerio de Educación Pública (MEP) avanza con planes como el Programa Nacional de Tecnologías Móviles que integra dotación y uso de TIC dentro del aula, con los procesos de enseñanza-aprendizaje (Programa Estado de la Nación, 2015). Las destrezas para el manejo de tecnologías educativas son una necesidad de estudiantes universitarios, más si estos se están formando para ejercer como futuros educadores y educadoras.

La metodología de trabajo que fundamentó el uso de los blogs como herramienta tecnológica se basa en dos principios: la divulgación de información en la era del conocimiento y aprender a aprender como herramienta cognitiva. El objetivo de este estudio fue valorar el uso del blog como herramienta pedagógica en el curso "Educación para la salud y la calidad de vida" desde las habilidades tecnológicas, las capacidades técnicas y la estrategia de evaluación, según el criterio de las estudiantes.

\section{La educación en la sociedad del conocimiento}

Vivimos una época donde el saber de las aulas no debe estar "encerrado en entregas» que sirven únicamente para medir el conocimiento adquirido en un curso. El estudiante participa de procesos de formación sin socializar sus aprendizajes con otras personas. Este debe estar en capacidad de producir y pensarse hacia ese mundo de información, aportando criterios y contenidos, con respaldo, sobre la materia propia de su disciplina y su formación profesional. Actualmente el conocimiento se produce en las redes de información, por tanto el estudiante debe ser capaz de integrarse en estas redes, seleccionar y producir conocimientos con cualidad académica para una sociedad en la cual su principal fuente de información es el Internet (Jonassen, 2013). En este sentido, el blog resultaría en un ejercicio para producir y crear no hacia adentro, sino hacia afuera de la universidad y al mismo tiempo hacia el curso del cual participa.

El segundo principio aprender a aprender se refiere a que la tecnología, más allá de ser utilizada para aprender en sí misma (por ejemplo, aprender a usar un blog como herramienta), es el medio por el cual los estudiantes expresan el conocimiento que poseen, adquieren y definen como relevante para su ejercicio profesional (Jonassen, 1995). Trascender el uso de la tecnología no solo con fines de aprendizaje per se; sino como una práctica desde el recurso.

El principio anterior se apoya en la teoría del Conectivismo (Siemens, 2005), por la cual el conocimiento es la acción de crear conexiones entre conjuntos especializados de información que permiten adquirir nuevos saberes. Es fundamental distinguir la información importante, por lo cual la capacidad crítica y la toma de decisiones se convierten en ejes centrales de la selección al navegar en el mar de información que se halla en la red. 
El blog como herramienta cognitiva facilita los procesos de aprendizaje y permite al estudiante organizar, interpretar, trabajar y representar sus conocimientos haciendo uso de recursos digitales, imágenes, mapas, ideas, entre otros. El estudiante al aprender a aprender de las fuentes, de la herramienta, de la interlocución en el espacio del curso, del contacto con el mundo que desborda informaciones sobre muchos temas, adquiere la habilidad reflexiva para resolver situaciones y producir conocimiento.

En el caso analizado en particular, se trata de la habilidad de seleccionar fuentes sobre temas pertinentes de salud en la dinámica escolar y apropiarse de estos para brindar información relevante en la Web, como señalan Martín y Moreno (2007): "Cuanto más eficaces se sientan los estudiantes, cuanto más confíen en que pueden influir en el futuro, más altas serán las metas que se propondrán y mayor su compromiso con ellas." ( p. 31).

\section{El blog como herramienta educativa de la WEB 2.0}

Con el amplio desarrollo que ha vivido el Internet a nivel global desde su nacimiento, la Web 2.0 se ha convertido en un instrumento de trabajo que apoya la educación. Se entiende la Web 2.0 como el espacio en Internet cuyas cualidades técnicas facilitan procesos de participación y colaboración donde el centro es el usuario como eje de la producción de contenidos.

Las plataformas permiten personalizar, enlazar y compartir la información de forma sencilla con las personas (Greenhow, Robelia, y Hughes, 2009). Las actuales capacidades de la Web 2.0 permiten crear redes de conocimiento entre personas y profesionales con intereses comunes, trabajar en colectivo y producir de forma simultánea e inmediata.

Dentro de las herramientas de la Web 2.0 se encuentra el blog. El blog es una herramienta cuyo uso se remonta a inicios de la década del 2000. En sus primeros años se relacionaba con experiencias de tipo «diario personal», donde las personas narraban partes de su vida privada en un espacio público en la red. Con la llegada de las plataformas como Blogger y WordPress, el acceso se masifica y sus capacidades técnicas, por lo fáciles de emplear, son accesibles a toda la población.

Los usos educativos más comunes se pueden clasificar en seis grandes categorías: 1) como un diario del aprendizaje; 2) como un portafolio personal de la experiencia de algún curso; 3) como un espacio para expresar las emociones; 4) para interactuar y comunicarse entre personas o grupos; 5) como una herramienta de evaluación y 6) como un repositorio de trabajos (Wee, Sim, y Hew, 2010).

Una experiencia docente de la web 2.0 mediante el uso de blogs con estudiantes de educación de la Universidad

54. Nacional, Heredia
Otras investigaciones más recientes en educación han profundizado en los aspectos evaluativos, y han señalado que al usar un blog se promueven destrezas y aprendizajes, ya que posee elementos creativos que incentivan y potencian nuevas habilidades tecnológicas y así permiten participar en procesos de 
aprendizaje cognitivos y metacognitivos (Chu, Chan y Tiwari, 2012; Neira-Piñeiro, 2015).

En el ámbito de habla español, se han llevado a cabo en los últimos diez años varias investigaciones donde mediaba el uso de blogs en el ámbito universitario (Aznar Cuadrado y Soto Carballo, 2010; Cabero Almenara, López Meneses y Ballesteros Regaña, 2009; Gewerc Barujel, 2005; López Meneses y Ballesteros Regaña, 2008; Molina, Antolín Jimeno, Pérez-Samaniego, Devís-Devís, y Villamón, 2013). Trabajos que muestran resultados positivos en el uso de los blogs, donde se ha potenciado el trabajo colaborativo, las bitácoras de campo, la promoción del aprendizaje autónomo y los repositorios. Es importante señalar que los estudios citados se han llevado a cabo temporalmente en distintos momentos (del 2006 a la fecha), lo cual los ha hecho variar tanto en capacidades técnicas como en el grado de experimentación.

En muchos casos, las investigaciones estaban principalmente centradas en el conocimiento mismo de la herramienta del blog, y no en la producción de contenidos vinculados a alguna asignatura por medio del blog. La principal problemática que han experimentado los blogs como recurso educativo se relaciona con la evaluación de los aprendizajes y la falta o ausencia de normas claras, o normas muy laxas, que no han logrado potenciar el recurso. A pesar de estos estudios, considerando la gran cantidad de instituciones de educación superior que existen hoy día, los blogs no son una herramienta común en la educación universitaria (González y García, 2010).

Otras investigaciones sobre el uso de los blogs en Latinoamérica han mostrado resultados positivos como herramienta para la redacción de ensayos académicos (Reyes Angona, Fernández-Cárdenas y Martínez Martínez, 2013). En Costa Rica, la investigación de Palma Villegas y Piñeiro Ruiz (2009) coincide con resultados favorables; no obstante, esta experiencia estaba centrada en el uso mismo de la herramienta del blog como instrumento de aprendizaje, no aplicado al conocimiento de una disciplina mediada a través del blog.

De las investigaciones se desprende que los blogs dan resultado en procesos educativos innovadores, mayormente positivos; sin embargo, en la mayoría de las investigaciones identificadas no se han logrado cambios significativos, ni en las formas de evaluación, ni en el currículo universitario; por el contrario, se han evidenciado las contradicciones curriculares de los sistemas educativos.

\section{La condición de género frente al uso de tecnologías}

El ejercicio de la profesión docente en Costa Rica ha sido mayoritariamente un oficio femenino (Programa Estado de la Nación, 2008). De acuerdo con el Banco Mundial, en el 2014 las mujeres representaban 80\% del cuerpo docente de primaria en el país (The World Bank, 2016). Por tanto, el tema del género y las tecnologías debe abordarse con interés, dado que la formación de niños y niñas se encuentra mayormente bajo la tutela de mujeres. Por esta razón, el tema de 
competencias tecnológicas docentes para el ejercicio de la enseñanza es de especial interés en el país (Programa Estado de la Nación, 2013).

Hay una postura que indica que la relación entre género y TIC es desigual debido a la falta de acceso, condiciones sociales, económicas o políticas, y no intrínsecamente por la condición de ser hombre o mujer (Hillbert, 2011). Una segunda postura, no excluyente, hace énfasis en la brecha digital entre hombres y mujeres. La brecha presenta asidero en los procesos de socialización, padres y escuela, donde las mujeres son percibidas con menores destrezas tecnológicas, lo cual muestra un impacto negativo que provoca mayores niveles de ansiedad e inseguridad al relacionarse con las TIC (Cooper, 2006). Los niveles de ansiedad han sido valorados frente al uso de las computadoras y, en todos los casos, las mujeres siempre manifiestan niveles más altos (Broos, 2005). Ambos planteamientos son debates abiertos. Estos estudios han estado principalmente asociados al manejo de la computadora como tal, y ante esto, se debería explorar cómo las nuevas tecnologías móviles y el acceso a las mismas podrían, o no, estar cambiando ambos panoramas.

No obstante, se ha comprobado que existen diferencias entre estudiantes universitarios hombres y mujeres y la percepción sobre el uso de herramientas Web 2.0. En el caso particular, las mujeres igualmente sobrellevaron niveles más altos de ansiedad, lo que podría deberse en principio a que las herramientas no son empleadas exclusivamente con fines académicos, no son por sí mismas pedagógicas (Huang, Hood, y Yoo, 2013).

En la región latinoamericana existen pocas investigaciones bajo un enfoque de género y TIC, la investigación de Martínez Martínez y Heredia Escorza (2010) mostró un mejor desempeño académico de las mujeres en relación con los hombres al hacer uso de computadoras y los proyectores en las aulas; no obstante, esta muestra fue pequeña y se señala la importancia de ampliar el estudio.

En la sociedad actual aún persisten diferencias de género en relación con el uso de las $\mathrm{TIC}$, diferencias que se alimentan de los estereotipos y prejuicios que se han construido, y que se acrecientan por desigualdades estructurales como lo son la etnia, la edad, el género, la condición socioeconómica y cultural (Bonder, 2008). Este testudio busca mostrar si el ejercicio llevado a cabo por mujeres, futuras educadoras, mediante el uso del blog como una herramienta de la Web 2.0 fue desde su óptica efectivo, y cuáles han sido los aspectos positivos y negativos de su implementación.

\section{Dinámica y evaluación del curso}

La herramienta se aplicó en el curso Educación para la salud y la calidad de la vida, forma parte del área de Desarrollo humano integral y su objetivo es "comprender, analizar, interpretar y problematizar las condiciones de la salud humana, la calidad de vida y su relación con aspectos fundamentales de la educación, el sistema educativo y su vida personal" (Programa del curso entregado a los estudiantes).

Una experiencia docente de la web 2.0 mediante el uso de blogs con estudiantes de educación de la Universidad

56. Nacional, Heredia 
La estrategia pedagógica relacionada con el uso de los blogs se estableció desde inicio del semestre. La segunda semana del curso se impartió una inducción sobre el uso del blog utilizando WordPress y Blogger; sin embargo, la escogencia de la plataforma era libre.

Cada estudiante debía redactar de cuatro a cinco entradas en el blog, las cuales se programaron una vez cada cuatro semanas, así como una sistematización individual, entre 500 y 1000 palabras, que incluyera al menos alguna de las lecturas vistas en ese periodo del curso. Adicionalmente, como parte de las asignaciones del curso, los estudiantes asistirían a una conferencia de libre elección durante el semestre, vinculada a un tema de salud para luego reportarla en el blog.

Se incentivó el uso de mapas conceptuales y el complementar las entradas con imágenes y videos. Los estudiantes gozaron de completa libertad para personalizar el nombre de su blog, colores, letras, fuentes, e inclusive la plataforma web que utilizaron. Para las entradas se debía respetar normas académicas establecidas en la universidad. No se permitiría el plagio, para evitar eso se solicitó incluir citas y referencias en el formato APA. También se aplicó una rúbrica de evaluación por medio de la cual se hizo retroalimentación de forma individual con su respectiva evaluación.

Se promovió una redacción enfocada hacia el grupo meta de educación y con información relacionada con alguno de los temas abarcados. A partir de esto, se les dio a los estudiantes el siguiente problema: si fuesen un padre o madre de un niño o niña en edad escolar que estuviera buscando información sobre los temas abordados en la asignatura, ¿qué información concisa, fundamentada y accesible, le podrían brindar mis intervenciones? Partiendo de esta premisa fueron realizadas las intervenciones en estos espacios.

Se debe aclarar que la retroalimentación de este curso no se realizó en la misma plataforma del blog, sino por medio de la rúbrica de evaluación de forma electrónica en el correo de cada estudiante.

\section{Método}

El estudio se desarrolló desde un enfoque cuantitativo-descriptivo con una muestra a conveniencia (McMillan y Schumacher, 2010). El carácter cuantitativo de este trabajo, como señala Muñoz Rocha "privilegia la información o los datos numéricos" (2015, p. 97), con el fin de presentar y exponer los aspectos más significativos de la experiencia del uso de los blogs.

La recolección de información se realizó a través un cuestionario de preguntas cerradas y dos preguntas abiertas donde los participantes manifestaron todas las posibles ventajas y desventajas del uso de los blogs.

La primera parte del cuestionario recolectaba información general para entender las características demográficas de los participantes, así como sus actitudes 
hacia la tecnología. La segunda parte consistía en escalas de valoración tipo Likert (Tymms, 2012), donde los informantes manifestaban cómo consideraron la experiencia en el uso de blogs.

El cuestionario fue autoadministrado mediante una plataforma en línea haciendo uso de un script de LimeSurvey en una página web habilitada solo para este fin. Las particularidades de los cuestionarios en línea es que facilitan la recolección y procesamiento de la información, asegurando una alta fidelidad de los datos (Hewson, Vogel, y Laurent, 2016). La información recolectada fue procesada usando el software SPSS para el análisis de datos y la construcción de cuadros y figuras gráficas.

En el estudio participaron 41 estudiantes, 20 correspondientes al año 2014 y 21 estudiantes del año 2015. Los participantes son mayoritariamente del sexo femenino ( $n=39)$, masculinos $(n=2)$. La edad promedio de las estudiantes fue 20,9 años. Con relación a su aproximación a la tecnología, las participantes afirman haber utilizado en promedio Internet por primera vez a los 12 años de edad ( $\mathrm{DS}=4,1$ años). El $90 \%$ de las participantes afirmaron no haber utilizado nunca antes la herramienta del blog.

Tabla 1: Características de las participantes

\begin{tabular}{llc}
\hline Condición & & $\%$ \\
\hline SEXO & Femenino & 95,1 \\
& Masculino & 4,87 \\
Nivel de Carrera & Primer año & 2.4 \\
& Segundo año & 85.4 \\
& Tercer año & 7.3 \\
& Cuarto año & 4.9 \\
& Quinto año & 2.4 \\
Colegio de procedencia & Público Urbano & 43.9 \\
& Otro & 24.4 \\
& Público Rural & 14.6 \\
& Técnico Profesional Público & 7.3 \\
& Privado Urbano & 7.3 \\
& Privado Rural & 2.4 \\
\hline
\end{tabular}

Fuente: elaboración propia con base en el instrumento

La edad y el nivel de carrera cursado por los participantes no presentaron variaciones significativas. La mayoría de los participantes provenía del sistema educativo público rural (14.6\%) o urbano (43.9\%). Otras opciones de colegio

Una experiencia docente de la web 2.0 mediante el uso de blogs con estudiantes de educación de la Universidad

58. Nacional, Heredia que se presentaron fueron bachillerato en educación diversificada por madurez, colegios nocturnos, o colegios subvencionados (semiprivados). 


\section{Resultados}

La mayoría de las estudiantes se autodefine, según el uso de las TIC, como personas con una capacidad media $46,35 \%$, y con tendencia alta $29,3 \%$. Ver la Gráfico 1.

Gráfico 1. Autodefinición de las estudiantes de sus capacidades para el uso de TIC

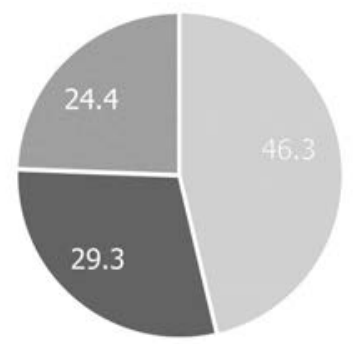

$$
\begin{aligned}
& \text { - Media } \\
& \text { - Alta } \\
& \text { - Baja }
\end{aligned}
$$

Gráfico 2. Hardware utilizado para elaborar las entradas en el blog.

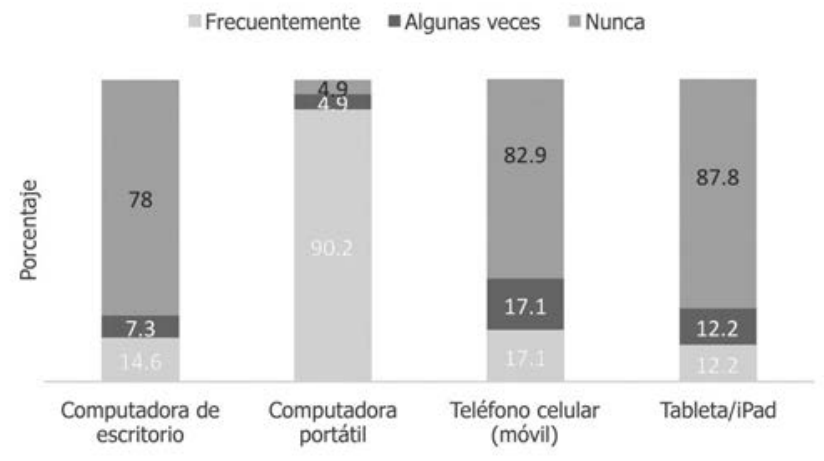

Llama la atención que, según muestra la Gráfico 2, de las opciones de dispositivos la principal herramienta utilizada para trabajar los blogs fue la computadora portátil. Otras herramientas tecnológicas como el celular, la tableta o la computadora de escritorio no fueron predominantes; sin embargo, tanto el celular como la tableta estaban por encima de la computadora de escritorio. Se debe resaltar el papel que las tecnologías portátiles (computadoras, celulares y tabletas) juegan en la producción de nuevos conocimientos, tareas y trabajos. En el futuro se debe valorar si esto se debe a la falta de acceso a computadoras de escritorio o si es preferencia personal.

Gráfico 3. Tiempo en relación con la producción de otros medios para elaborar trabajos. 


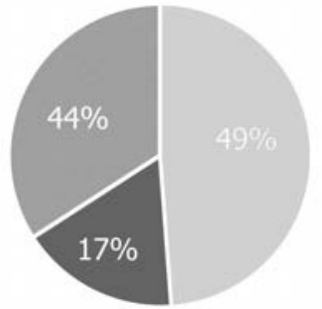

" El mismo tiempo que hacer el trabajo en

formato impreso

- Más tiempo que hacer un trabajo en formato impreso

- Menos tiempo que hacer un trabajo en formato impreso

Los estudiantes consideraron que el tiempo destinado a hacer los trabajos utilizando los blogs, era similar al que hubieran destinado a un trabajo en formato impreso; sin embargo, un porcentaje importante (34\%) piensa que fue menor, y es relativamente bajo (17\%) el número que cree que debió destinar más tiempo a la labor.

Gráfico 4. Percepción sobre aspectos académicos y evaluativos del uso del blog.

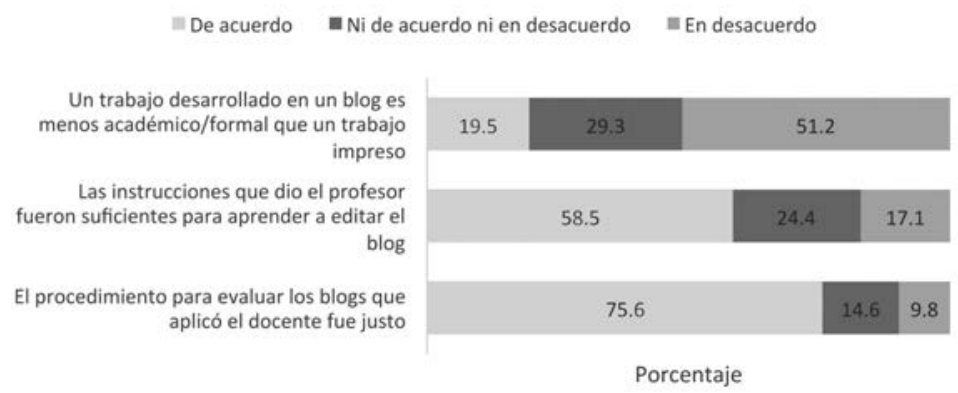

De acuerdo con la Gráfico 4, una mayoría (75\%) de los participantes estuvo de acuerdo con que se aplicó un proceso justo de evaluación. Un porcentaje menor (58\%) considera que las instrucciones fueron suficientes, sin embargo, algunas estudiantes (25\%) no estuvieron ni de acuerdo ni en desacuerdo. La principal divergencia se presenta en la condición académica del blog, donde un 51,2\% no considera que sea menos académico, pero sumando las otras dos opciones de respuesta casi la mitad no está convencida de su carácter académico.

Gráfico 5. Percepción sobre aspectos técnicos y tecnológicos sobre el uso del blog.

Una experiencia docente de la web 2.0 mediante el uso de blogs con estudiantes de educación de la Universidad

60. Nacional, Heredia

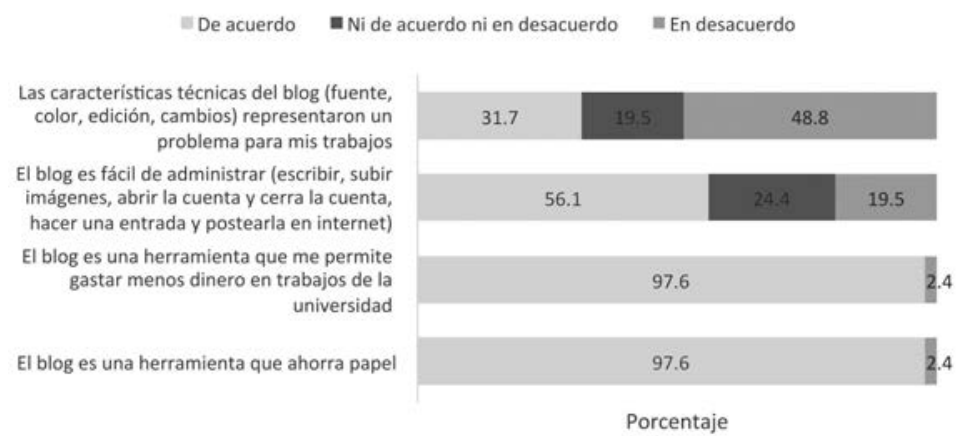


De acuerdo a la Gráfico 5, el blog destaca como una herramienta de aprendizaje que permite menos gasto y ahorra papel (97\%). Así mismo, el blog resultó fácil para un $56 \%$ de las participantes, cuyas características técnicas no fueron un problema para el $48 \%$ de las estudiantes.

Figura 6. Actitudes y destrezas con relación al uso del blog.

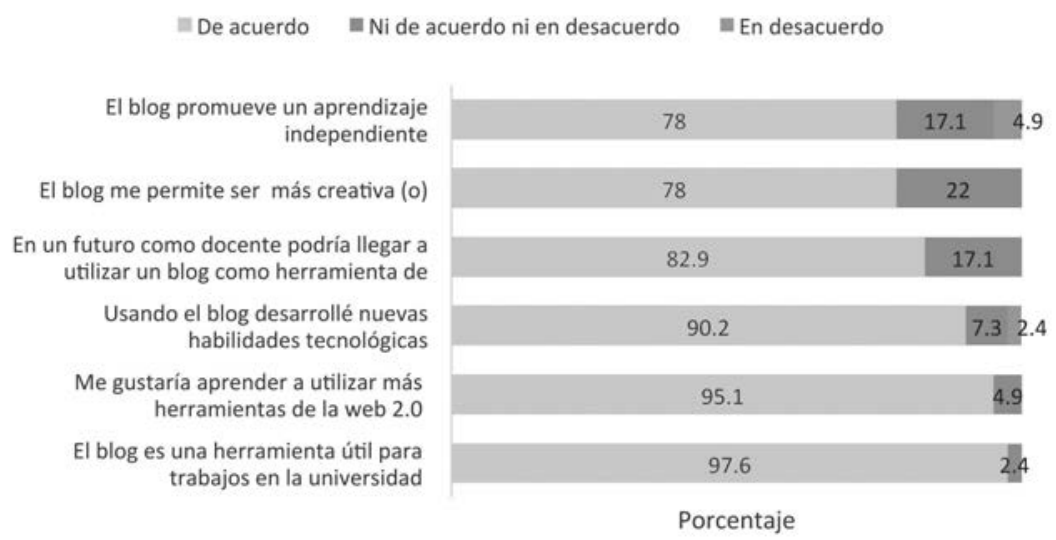

La Gráfico 6 muestra que la experiencia de utilizar el blog como herramienta para presentar y elaborar los trabajos del curso fue positiva. La mayoría (97\%) considera que el blog es una herramienta útil, y le gustaría aprender más sobre herramientas de la web 2.0 (95\%). Así mismo manifiestan haber desarrollado nuevas habilidades tecnológicas (90\%), permite la creatividad (78\%) y promueve un aprendizaje independiente (78\%). Un alto porcentaje (82\%) manifiesta que podría llegar a usar el blog como herramienta de trabajo.

En la Tabla 2 se muestra una selección de respuestas representativas elaboradas con base en la pregunta abierta mediante la cual se les consultó a los estudiantes, a partir de su experiencia, todas las posibles ventajas y desventajas del uso de los blogs. A partir de las respuestas se puede ampliar que la mayoría de las posibles desventajas que se suscitaron estaban relacionadas con la poca instrucción - guía recibida al inicio del curso para desarrollar sus trabajos. También surgió la necesidad de poseer Internet para poder subir sus entradas. Posibles fallos en el uso de la herramienta, las capacidades personales o habilidades y las limitaciones o instrucciones que estableció el profesor al inicio del curso, como por ejemplo, la cantidad de entradas o la cantidad de palabras. 
Tabla 2. Ventajas y desventajas del uso de la herramienta de blog.

\begin{tabular}{l} 
Ventajas \\
\hline Conocimiento en redes tecnológicas que eran \\
ajeno a mi persona, ventajas económicas se \\
redujo el gasto de tinta y papel, fácil acceso \\
y una forma más rápida para luego buscar la \\
información".
\end{tabular}

"La forma de entregar los trabajos era más dinámica, sin tener que utilizar el formato de todos, se podía usar la creatividad en el uso de mapas conceptuales. El no tener que estar imprimiendo".

"Se comparte con más personas, a la hora de elaborar un trabajo se está consciente de que puede ser leído por diferentes personas y no solamente por el profesor o profesora del curso, estas personas pueden dar aportes, sugerencias o comentarios que pueden ser fructíferos para un crecimiento y nuevos conocimientos".

"Podría ser una forma en la que algunas personas podrían descubrir otras habilidades relacionadas a este tema, tanto para la tecnología como para escribir sobre temas de interés y actuales".

"Una de las ventajas que encuentro es que al ser un blog público otras personas lo pueden leer e informarse sobre los temas relevantes escritos en él. La mayoría de reflexiones y trabajos realizados en estos tres años de universidad se han quedado guardados en $\mathrm{mi}$ computadora a excepción de los subidos al blog en este curso por lo que me parece muy interesante la estrategia. Es un medio además para compartir conocimientos y conocer otras opiniones".

"las devoluciones de los trabajos fue bastante clara y oportuna, siento que facilitaba el trabajo de ambas partes".

\section{Desventajas}

Poca capacitación en el manejo del programa".

"Desconocimientos de procedimientos y herramientas para lograr hacer un trabajo más completo y profesional".

"La entrada es muy restringida por el profesor( tiene que ser de 750 palabras mínimo por obligación y de la metodología que diga el profesor) esto niega muchas ideas que pueda tener el estudiante".

"Es una estrategia interesante al principio pero no deberían de ser 5 entradas, ya que se vuelve tedioso y un poco rutinario".

"Al saber que otras personas aparte del profesor lo pueden leer hace que se medite demasiado en lo que se va a escribir pero por lo demás me gustó la experiencia".

"Que no todas las personas tienen gusto por descubrir cosas como estas y por más que intenten, se les podría dificultar el uso de estas herramientas".

"Me dedica un poco más de tiempo, debido a que al colocarse en Internet y tener acceso otras personas, el documento debía llevar una mejor calidad de ortografía y redacción, por lo que tuve que indagar e investigar bastante".

"No poseer Internet en la casa, pero considero que actualmente no debería ser un problema" "El constante uso de una computadora, el no saber utilizarla bien y menos para subir un documento el tener que gastar más tiempo en la compu".
Una experiencia docente de la web 2.0 mediante el uso de blogs con estudiantes de educación de la Universidad

62. Nacional, Heredia
Dentro de las ventajas destacan el haber adquirido nuevas habilidades y destrezas tecnológicas, no tener que imprimir los trabajos, no ser un gasto económico de más, potenciar la creatividad, socializar los trabajos de la universidad con otros grupos, compartir conocimiento y conocer el blog de otras personas.

\section{Discusión de resultados}

Se solicitó a las estudiantes valorar de 0 a 10 (siendo diez el valor máximo) otorgar una calificación a la experiencia de utilización del blog como herramienta del curso. El puntaje promedio otorgado por las participantes fue de 8,34 puntos. 
Sobre el uso del blog. Las percepciones de uso del blog como herramienta de evaluación y aprendizaje fueron positivas. La mayoría de las estudiantes consideró haber desarrollado nuevas habilidades tecnológicas, destacó la creatividad, la personalización y el compromiso de saber que, al escribir, podía ser leída por una persona en cualquier parte del mundo. El blog resultó ser una herramienta práctica para la presentación de los trabajos.

Como parte de los aspectos negativos se señalan, el acceso a Internet, la poca capacitación que brindó el docente al inicio del curso sobre el manejo de la herramienta, así como las limitaciones impuestas por las instrucciones en relación con la cantidad de palabras, y algo que no es en sí misma una limitación, pero que representa un elemento de cuidado, la percepción en algunos casos negativa hacia ser leído o visto por personas dentro del mismo curso o fuera de él, lo que pudo jugar algún papel en el desempeño. Esto es un punto recurrente a favor y en contra, que podría ampliarse en futuras investigaciones.

Los estudiantes no estaban completamente seguros de si su trabajo resultaba en un producto "menos académico" por haber sido desarrollado en un blog, lo que muestra que existe poca familiaridad con nuevas formas de producción y creación de conocimiento académico mediante Internet.

Sobre el género. Casi todas las participantes (90\%) no habían utilizado nunca la herramienta del blog. Llama la atención de la poca familiaridad que las estudiantes mostraban, pese a su formación anterior a la universidad y universitaria, sobre distintas herramientas tecnológicas, en especial, las herramientas de la Web 2.0.

Lo que han apuntado otras investigaciones es que, al igual que con otras herramientas tecnológicas, el desarrollo de actividades en línea con la Web 2.0 genera algún tipo de ansiedad en las mujeres, por ejemplo, para colaborar o para llevar a cabo actividades como wikis, o tan solo el hecho de comentar y leer a otras compañeras crea igualmente niveles de ansiedad en las estudiantes (Huang et al., 2013).

El principal hallazgo de este estudio en relación con el género es que, a pesar de las barreras reconocidas sobre el tema y su relación con las tecnologías, las estudiantes mostraron anuencia al manejo de la herramienta tecnológica, misma que no representó una barrera por su condición femenina. No obstante, los resultados de esta investigación no midieron la ansiedad como un factor, los resultados positivos de este estudio señalan que, aunque aún se debe trabajar, las brechas de género y tecnología podrían estarse reduciendo gracias al acceso masivo a nuevos dispositivos tecnológicos portables, que familiarizan de forma temprana tanto a hombres como a mujeres (Top, Yukselturk y Cakir, 201 1). Hipótesis que debe comprobarse en nuevas investigaciones.

Implicaciones institucionales. Este año, las universidades estatales, y entre ellas la Universidad Nacional, han declarado Año de la Madre Tierra (UNA, 2016), 
impulsando acciones en torno a formas más ecológicas de convivencia, por ende, pedagogías y acciones educativas que ayuden a mitigar el impacto que los seres humanos provocan en el planeta. Los blogs han destacado como una modalidad que disminuye la impresión de textos, así como el consumo innecesario de papel y tintas. Al mismo tiempo, la disposición y la tendencia al uso de dispositivos móviles abren la posibilidad de potenciar aprendizajes ubicuos, y de integrar las mismas herramientas como los blogs, las wikis o el microbloging.

Asimismo, la investigación evidencia que se pueden llevar a cabo procesos académicos de formación haciendo uso de tecnologías disponibles en Internet sin costo alguno. Lo anterior plantea el desafío de establecer con mayor claridad, a lo largo de la formación profesional e inclusive en los reglamentos, el uso de recursos digitales para variar los métodos tradicionales de enseñanza y evaluación de los aprendizajes, los cuales potencien distintas habilidades y capacidades en los estudiantes.

Finalmente, se evidencia que al implementar estas experiencias debe existir acompañamiento docente (instrucción, mediación, evaluación, retroalimentación) para que resulten en procesos de aprendizaje efectivos. En otras experiencias de investigación, al haber mucha "libertad" se derivó en una menor calidad de los blogs, poco entendimiento sobre las expectativas educativas o trabajos con diferencias importantes en contenido y calidad (Molina Aventosa, Valenciano Valcárcel y Valencia-Peris, 2015).

Vacíos y líneas de investigación a futuro. Como vacíos de información y posibles nuevas líneas de análisis está pendiente valorar por qué los estudiantes no comentaron frecuentemente el trabajo de otros. También sería importante establecer contacto para saber si posterior a esta experiencia se presenta continuidad o abandono en el uso de estas herramientas. Es importante revisar y evaluar las capacidades y preferencias tecnológicas de las futuras docentes en concordancia con su perfil profesional a futuro.

Por otra parte, un análisis igualmente pendiente es realizar un estudio del contenido generado por medio de estos espacios y la calidad de los materiales y las intervenciones que se llevaron a cabo. Habrá que valorar el porqué no es común el uso de celulares y/o tabletas y computadoras de escritorio como herramientas para crear conocimiento, explorar y valorar, o cómo potenciar estos nuevos recursos, aun considerando que los dispositivos móviles (si se consideran las computadoras portátiles) son las herramientas de uso predominante.

Una experiencia docente de la web 2.0 mediante el uso de blogs con estudiantes de educación de la Universidad

64. Nacional, Heredia

\section{Conclusión}

En términos de las TIC, las herramientas han cambiado en los últimos años. Recursos que hace diez años eran comunes hoy o no lo son, surgen nuevas plataformas tecnológicas que facilitan la enseñanza. El aprendizaje debe estar basado en despertar inquietudes, en la apropiación del conocimiento, la exploración, la 
duda, el aprender a aprender. Este trabajo muestra que los estudiantes están en capacidad de usar herramientas tecnológicas de la Web 2.0 como el blog, las cuales potencian la creatividad, las habilidades tecnológicas y la cultura digital, sin perder la rigurosidad académica universitaria.

El principal valor de las herramientas de la Web 2.0 es la posibilidad de la colaboración, el trabajo colectivo. Un reto de herramientas como los blogs es el diseño de estrategias colaborativas más claras y productivas, cuyos productos sean valorados de distintas formas (Hsu, Ching, y Grabowski, 2014). De esta experiencia queda pendiente el integrar estrategias como el microb/oging, o la edición colaborativa de materiales de tipo audiovisual. Muchas de las actuales herramientas aún no han explotado (o al menos de la evidencia académica) todos los recursos que posibilitan.

El blog es una herramienta para crear textos, sustituye el papel tradicional e incorpora elementos como el hipertexto, el material audiovisual, la personalización, además de trascender la geografía y el tiempo. Como señala Vygotsky "El pensamiento no se expresa simplemente con palabras; llega a la existencia a través de ellas" (2014, p. 285), el fin último es crear conocimientos significativos durante el proceso de aprendizaje y, por qué no entonces, aprovechar todas las herramientas que ofrece la tecnología para este fin.

\section{Referencias}

Aznar Cuadrado, V., y Soto Carballo, J. (2010). Análisis de las aportaciones de los blogs educativos al logro de la competencia digital. Revista de Investigación en Educación, 7(7), 83-90. Recuperado de http://webs.uvigo.es/reined/ojs/index. php/reined/article/ view/81

Bonder, G. (2008). Juventud, Género \& TIC: Imaginarios en la construcción de la sociedad de la información en América Latina. Arbor, 184(733), 917-934. http://doi.org/ 10.3989/arbor.2008.i733.234

Broos, A. (2005). Gender and Information and Communication Technologies (ICT) Anxiety: Male Self-Assurance and Female Hesitation. CyberPsychology \& Behovior, 8(1), 21-31. http://doi.org/10.1089/cpb.2005.8.21

Cabero Almenara, J., López Meneses, E., y Ballesteros Regaña, C. (2009). Experiencias universitarias innovadoras con blogs para la mejora de la praxis educativa en el contexto europeo. Revista de Universidad y Sociedad del Conocimiento, 6(2), 1-14. Recuperado de http://rusc.uoc.edu/index.php/rusc/article/view/v6n2 cabero_etal/ v6n2_cabero

Castells, M. (2006). Teoría de la Sociedad Red. En M. Castells (Ed.), La Sociedad Red: Una Visión Global (pp. 27-78). Madrid: Alianza Editorial.

Chu, S. K. W., Chan, C. K. K., y Tiwari, A. F. Y. (2012). Using blogs to support learning during internship. Computers \& Education, 58(3), 989-1000. http://doi.org/10.1016/ j.compedu. 2011.08 .027

Cooper, J. (2006). The digital divide: the special case of gender. Journal of Computer Assisted Learning , 22(5), 320-334. http://doi.org/10. 111 1/j. 1365-2729.2006.00185.x

Gewerc Barujel, A. (2005). El uso de weblogs en la docencia universitaria. RELATEC: Revista 
Latinoamericana de Tecnología Educativa, 4(1), 9-24. Recuperado de http:// relatec.unex.es/article/view/173/163

González, R., y García, F. (2010). Propuesta de un modelo de medición del desarrollo de los blogs educativos. Una aplicación empírica el sistema educativo español. Revista de Formación e Innovación Educativa Universitaria, 3(1), 8-20. Recuperado de http://refiedu.webs.uvigo.es/Refiedu/Vol3_1/REFIEDU_3_1_2.pdf

Greenhow, C., Robelia, B., y Hughes, J. E. (2009). Learning, Teaching, and Scholarship in a Digital Age: Web 2.0 and Classroom Research: What Path Should We Take Now? Educational Researcher, 38(4), 246-259. http://doi. org/10.3102/0013189X09336671

Hewson, C., Vogel, C., y Laurent, D. (2016). Internet Research Methods (2.a ed.). London: SAGE Publications Ltd.

Hilbert, M. (201 1). Digital gender divide or technologically empowered women in developing countries? A typical case of lies, damned lies, and statistics. Women's Studies International Forum, 34(6), 479-489. http://doi.org/10.1016/j.wsif.201 1.07.001

Hsu, Y., Ching, Y., y Grabowski, B. L. (2014). Web 2.0 Applications and Practices for Learning Through Collaboration. En J. M. Spector, M. D. Merrill, J. Elen, y M. J. Bishop (Eds.), Handbook of Research on Educational Communications and Technology (pp. 747-758). New York, NY: Springer New York. http://doi.org/ 10.1007/978-1-4614$3185-5$

Huang, W.-H. D., Hood, D. W., y Yoo, S. J. (2013). Gender divide and acceptance of collaborative Web 2.0 applications for learning in higher education. The Internet and Higher Education, 16, 57-65. http://doi.org/10.1016/j.ineduc.2012.02.001

Jonassen, D. H. (1995). Computers as cognitive tools: Learningwith technology, notfrom technology. Journal of Computing in Higher Education, 6(2), 40-73. http://doi. org/10.1007/BF02941038

Jonassen, D. H. (2013). Transforming Learning with Technology. En The Nature of Technology (pp. 101-110). Rotterdam: SensePublishers. http://doi.org/10.1007/978-94-6209269-3 7

Kalaš, I. (2010). Recognizing the potential of ICT in early childhood education. Moscow, Russian Federation: UNESCO Institute for Information Technologies in Education. Recuperado de http://unesdoc.unesco.org/images/0019/001904/190433e.pdf

López Meneses, E., y Ballesteros Regaña, C. (2008). Caminando hacia el software social: una experiencia universitaria con blogs. Pixel-Bit. Revista de Medios y Educación, (32), 67-82. Recuperado de http://acdc.sav.us.es/ojs/index.php/pixelbit/article/view/ 680

Martín, E., y Moreno, A. (2007). Competencia para aprender a aprender. Madrid: Alianza Editorial.

Martínez Martínez, R., y Heredia Escorza, Y. (2010). Tecnología educativa en el salón de clase. Revista Mexicana de Investigación Educativa, 15(45), 371-390. Recuperado de http://www.comie.org.mx/v1/revista/portal.php?idm =es\&sec $=$ SC03\&\&sub=SBB\& criterio $=$ ART 45003

McMillan, J., y Schumacher, S. (2010). Investigación Educativa (5.a ed.). Madrid: Pearson/

Una experiencia docente de la web 2.0 mediante el uso de blogs con estudiantes de educación de la Universidad 66. Nacional, Heredia

\section{Addison Wesley.}

Molina Aventosa, P., Valenciano Valcárcel, J., y Valencia-Peris, A. (2015). Los blogs como entornos virtuales de enseñanza y aprendizaje en Educación Superior. Revista Complutense de Educación, 26 (Especial), 15-31. http://doi.org/10.5209/rev_ RCED.2015.v26.43791 
Molina, P., Antolín Jimeno, L., Pérez-Samaniego, V., Devís-Devís, J., y Villamón, M. (2013). Uso de blogs y evaluación continua del aprendizaje del alumnado universitario. EDUTEC. Revista Electrónica de Tecnología Educativa, 43(Marzo). Recuperado de http://www.edutec.es/revista/index.php/edutec-e/article/view/335

Muñoz Rocha, C. (2015). Metodología de la Investigación (1.a ed.). México, D.F.: Oxford University Press.

Neira-Piñeiro, M. del R. (2015). Reading and writing about literature on the Internet. Two innovative experiences with blogs in higher education. Innovations in Education and Teaching International, 52(5), 546-557. http://doi.org/10.1080/14703297.2014. 900452

Palma Villegas, C., y Piñeiro Ruiz, M. (2009). La implementación del blog en la Educación Superior: el caso del curso tecnología educativa y recursos para el aprendizaje. Inter Sedes, 10(18), 142-153. Recuperado de http://www.redalyc.org/pdf/666/ 66618385005.pdf

Programa Estado de la Nación. (2008). Segundo Informe Estado de la Educación. San José, Costa Rica.

Programa Estado de la Nación. (2013). Cuarto Informe Estado de la Educación. San José, Costa Rica.

Programa Estado de la Nación. (2015). Quinto Informe Estado de la Educación. San José, Costa Rica.

Reyes Angona, S., Fernández-Cárdenas, J. M., y Martínez Martínez, R. (2013). Comunidades de blogs para la escritura académica en la enseñanza superior: un caso de innovación educativa en México. Revista Mexicana de Investigación Educativa, 18(57), 507-535. Recuperado de http://www.comie.org.mx/v1/revista/ portal.php? $\mathrm{idm}=$ es\&sec $=$ SC03\&\&sub $=$ SBB\& $\&$ riterio $=$ ART 57008

Selwyn, N. (2014). La innovación digital en educación: implicaciones para estudiantes, profesores y escuelas. En J. Tarango y J. L. García Rodríguez (Eds.), Modelos e iniciativas en educacion media superior (pp. 241-265). Buenos Aires: Alfagrama Ediciones.

The World Bank. (2016). Costa Rica primary education, teachers (\% female). Recuperado de http://data. worldbank.org/indicator/SE.PRM.TCHR.FE.ZS?end=2013\&locations= CR\&name_desc $=$ true\&start $=2006$

Top, E., Yukselturk, E., y Cakir, R. (201 1). Gender and Web 2.0 technology awareness among ICT teachers. British Journal of Educational Technology, 42(5), E106-E109. http:// doi.org/10.1111/j.1467-8535.2011.01208.x

Tymms, P. (2012). Questionnaires. En J. Arthur, M. Waring, R. Coe, y L. V. Hedges (Eds.), Research Methods and Methodologies in Education (pp. 231-240). London: SAGE Publications Ltd.

UNA. (2016). Año de la UNA por la Madre Tierra. Recuperado de http://www. madretierra. una.ac.cr/

UNESCO. (2000). Marco de Acción de Dakar. Educación para Todos: cumplir nuestros compromisos comunes. Organización de las Naciones Unidas para la Educación, la Ciencia y la Cultura. Recuperado de http://unesdoc.unesco.org/ images/ 0012/001211/121147s.pdf

Vygotsky, L. (2014). Pensamiento y Lenguaje. Barcelona, España: Paidós.

Wee, J., Sim, S., y Hew, K. F. (2010). The use of weblogs in higher education settings : A review of empirical research. Educational Research Review, 5(2), 151-163. http:// doi.org/10.1016/j.edurev.2010.01.001 